ISSN 0258-7122

Bangladesh J. Agril. Res. 37(4): 607-616, December 2012

\title{
EFFECTS OF NITROGEN AND POTASSIUM ON GROWTH AND YIELD OF GLADIOLUS CORMS
}

\author{
F.N. KHAN ${ }^{1}$, M.M. RAHMAN ${ }^{2}$, A. J. M. S. KARIM ${ }^{2}$ \\ K. M. HOSSAIN ${ }^{3}$
}

\begin{abstract}
A study was conducted at the Floriculture Research Field of Horticulture Research Centre of Bangladesh Agricultural Research Institute (BARI) during the period from November 2006 to May 2008 to determine the optimum rate of $\mathrm{N}$ and $\mathrm{K}$ for better growth and yield of corm and cormel of gladiolus. The treatment combination $\mathrm{N}_{150} \mathrm{~K}_{200} \mathrm{~kg} /$ ha produced the longest plant $(42.1 \mathrm{~cm})$, the broadest leaf $(1.93 \mathrm{~cm})$, the maximum percentage of spikes $(88.1 \%)$, and corm $(97.6 \%)$, the heaviest and the largest corm $(19.5 \mathrm{~g}$ and $4.11 \mathrm{~cm}$, respectively), cent percent flowering sized corm, and the highest corm number and cormel yield (1,20,000 and $1.66 \mathrm{t} / \mathrm{ha}$, respectively). The corm produced from this treatment combination also showed better performances in the next year in respect of plant emergence (100\%), florets/spike (13.1), spike and rachis length $(82.2 \mathrm{~cm}$ and $45.4 \mathrm{~cm}$, respectively), flower stick weight $(57.1 \mathrm{~g})$ and percentage of flower sticks $(113 \%)$.
\end{abstract}

Keywords: Nitrogen, potassium, corm, cormel and gladiolus.

\section{Introduction}

Gladiolus is the second most popular flower in the world, especially from the commercial point of view. Gladiolus has great economic value and wide market in Bangladesh, however, the yield, quality of flower, and year round supply have not been achieved up to the mark. Lack of quality planting material is one of the causes behind it. Availability of planting materials is pre-requisite for commercial cultivation of gladiolus. As such, there is a great scope of establishing a commercial production of corm and cormel.

Production of healthy and vigorous corms and cormels depend on many factors, of which nutrient supply is an important one. Gladiolus requires nutrients throughout the period of growth, corm development, and flowering. So, application of suitable nutrients in an optimum amount is important. Gladiolus cormels responded better to heavy doses of fertilizers compared to corms (Mukhopadhaya, 1995). Increasing nitrogen (N) augmented plant growth, number of leaves/plant, spike length, and number of florets, spike (Shah et al.,

\footnotetext{
${ }^{1}$ Senior Scientific Officer, Floriculture Division (HRC), Bangladesh Agricultural Research Institute (BARI), Gazipur, ${ }^{2}$ Professor, Bangabandhu Sheikh Mujibur Rahman Agricultural University (BSMRAU), Gazipur; ${ }^{3}$ Ex-Principal Scientific Officer, Soil Science Division, BARI, Gazipur, Bangladesh
} 
1984). Due to $\mathrm{N}$ deficiency production of corms and cormels was affected and the plants could not utilize the stored food in the old corms properly (Mukhopadhyay, 1995). Potassium works as a catalyst in many biosynthetic reactions of the photosynthesis and the element $\mathrm{K}$ is involved in the synthesis of amino acids and gives resistance to plant against diseases. In the absence of adequate dose of $\mathrm{K}$, higher doses of $\mathrm{N}$ are not beneficial to flower quality due to nutritional imbalance (Mukhopadhyay, 1995). Nitrogen, phosphorus, and potassium greatly influence the growth, flower, and tuber production of tuberose. Nutrients were 4 levels of $\mathrm{N}(0,100,200$, and $300 \mathrm{~kg} \mathrm{~N} / \mathrm{ha}), 3$ levels of phosphorus $(0,45$, and $90 \mathrm{~kg} \mathrm{P} / \mathrm{ha})$, and 3 levels of potassium $(0,80$, and $160 \mathrm{~kg}$ $\mathrm{K} / \mathrm{ha}$ ) were tested on the tuberose. A positive impact of fertilizer combinations was observed (Sultana et al., 2006).

The growers do not have any recommended doses of chemical fertilizers for quality corm and cormel production. Even the flower producers multiply their seeds without applying any chemical fertilizers. As a result, they are deprived to get optimum sized corms and cormels for flower cultivation. So, there is a good scope of increasing the yield and vigorous corm and cormel production of gladiolus from cormel by the use of appropriate amount of $\mathrm{N}$ and $\mathrm{K}$ under the agro-ecological condition of Bangladesh. However, information regarding nutritional requirement for corm and cormel production from cormel in Bangladesh is lacking. Keeping these in views, the present study was undertaken to investigate the effects of $\mathrm{N}$ and $\mathrm{K}$ on growth, yield of corm, and cormel production of gladiolus from cormel and to study the effects of $\mathrm{N}$ and $\mathrm{K}$ on the quality of corms from cormels within short period.

\section{Materials and Method}

The experiment was carried out at Floriculture Research Field of Horticulture Research Centre, Bangladesh Agricultural Research Institute (BARI), Gazipur during November 2006 to May 2008. Medium sized (1.5-2.0 cm diameter) cormels of BARI Gladiolus-1 were used as planting materials. Nutrient status of initial soil is presented in Table 1 . There were 12 treatments where the first six treatments $\left(T_{1}\right.$ to $\left.T_{6}\right)$ contained $N$ levels at $0,50,100,150,200$, and $250 \mathrm{~kg} / \mathrm{ha}$ with a single dose of $\mathrm{K}(120 \mathrm{~kg} / \mathrm{ha})$ and treatments $\mathrm{T}_{7}$ to $\mathrm{T}_{11}$ had $\mathrm{K}$ levels at 0,40 , 80,160 , and $200 \mathrm{~kg} / \mathrm{ha}$ with a single dose of $\mathrm{N}(150 \mathrm{~kg} / \mathrm{ha})$. The treatment $\mathrm{T}_{12}$ was absolute control.

Table 1. Nutrient status of initial soil of experimental field.

\begin{tabular}{|c|c|c|c|c|c|c|c|c|c|c|c|c|c|}
\hline \multirow{2}{*}{ Year } & \multirow{2}{*}{$\mathrm{P}^{\mathrm{H}}$} & Total N & $\mathrm{OM}$ & $\mathrm{Ca}$ & $\mathrm{Mg}$ & K & $\mathrm{P}$ & $\mathrm{S}$ & B & $\mathrm{Cu}$ & $\mathrm{Fe}$ & $\mathrm{Mn}$ & $\mathrm{Zn}$ \\
\hline & & \multicolumn{2}{|c|}{$\%$} & \multicolumn{3}{|c|}{ Meq/100 g } & \multicolumn{7}{|c|}{$\mu \mathrm{g} / \mathrm{g}$} \\
\hline $2006-2007$ & 6.1 & 0.077 & 1.46 & 4.76 & 1.97 & 0.15 & 15 & 38 & 0.32 & 6.0 & 232 & 10 & 3.30 \\
\hline Critical level & & & & 2.0 & 0.8 & 0.2 & 14 & 14 & 0.2 & 1.0 & 10.0 & 5.0 & 2.0 \\
\hline
\end{tabular}

Source: Soil Science Division, BARI, Joydebpur, Gazipur 
The experimental field was prepared by deep ploughing. A common dose of cowdung (5 t/ha), TSP (50 kg P/ha), zinc sulphate ( $3 \mathrm{~kg} \mathrm{Zn/ha),} \mathrm{and} \mathrm{gypsum} \mathrm{(10}$ $\mathrm{kg} \mathrm{S} / \mathrm{ha}$ ) were used except for the control treatment. Different levels of $\mathrm{N}$ and $\mathrm{K}$ were used as per treatments. The entire amount of cowdung, TSP, MoP, zinc sulphate, and gypsum were applied at the time of final land preparation as basal and urea as a source of $\mathrm{N}$ was top-dressed in three equal splits at 15, 45, and 75 days after sowing.

The experiment was laid out in the Randomized Complete Block Design (RCBD) with three replications. The cormels were planted at $20 \mathrm{~cm} \times 15 \mathrm{~cm}$ spacing in unit plot of $1.2 \mathrm{~m} \times 1.05 \mathrm{~m}$. The cormels of BARI Gladiolus-1 were planted on 25 November 2006. Intercultural operations like weeding, watering were done as and when required. The cormel plants were not allowed to flower since it might reduce the production of corm and cormels. The spikes were cut off just after spike initiation to enhance quality corm and cormel production. Harvesting of corms and cormels were done only when leaves turned brown (Mukhopadhaya, 1995). After collection of data, the corms were stored in a perforated nylon bag at normal room temperature. These corms were planted on 9 December 2007 to observe the performance of flower production. Data on different growth and yield parameters were recorded and analyzed statistically.

\section{Results and Discussion}

\section{Effects of $\mathbf{N}$ and $\mathrm{K}$ on vegetative growth and spikes production of gladiolus}

Significant variations were observed on vegetative growth of gladiolus except leaf length due to the effect of $\mathrm{N}$ and $\mathrm{K}$ (Table 2).

Leaf length: Though leaf length did not show significant variations among the treatments, but the longest leaf $(36.6 \mathrm{~cm})$ was produced by $\mathrm{T}_{6}\left(\mathrm{~N}_{250} \mathrm{~K}_{120} \mathrm{~kg} / \mathrm{ha}\right)$ and the shortest leaf $(32.7 \mathrm{~cm})$ was recorded in control (Table 2).

Leaf breadth: The broadest leaf $(1.93 \mathrm{~cm})$ was produced by $\mathrm{T}_{11}\left(\mathrm{~N}_{150} \mathrm{~K}_{200} \mathrm{~kg} / \mathrm{ha}\right)$ which was statistically identical to $\mathrm{T}_{6}\left(\mathrm{~N}_{250} \mathrm{~K}_{120} \mathrm{~kg} / \mathrm{ha}\right)$. The narrowest leaf was recorded in control $(1.55 \mathrm{~cm})$ followed by a number of treatments like $T_{1}, T_{7}, T_{2}$, $\mathrm{T}_{3}, \mathrm{~T}_{8}, \mathrm{~T}_{4}$, and $\mathrm{T}_{9}$ (Table 2). 
Plant height: Plant height was significantly different among the treatments (Table 2). The longest plant $(42.1 \mathrm{~cm})$ was produced by the treatment $\mathrm{T}_{11}\left(\mathrm{~N}_{150}\right.$ $\mathrm{K}_{200} \mathrm{~kg} / \mathrm{ha}$ ) followed by $\mathrm{T}_{6}, \mathrm{~T}_{10}, \mathrm{~T}_{5}, \mathrm{~T}_{9}, \mathrm{~T}_{4}, \mathrm{~T}_{3}, \mathrm{~T}_{8}, \mathrm{~T}_{7}$, and $\mathrm{T}_{2}$. The shortest plant was produced by the treatment control $(38.4 \mathrm{~cm})$, which was statistically identical with $\mathrm{T}_{1}\left(\mathrm{~N}_{0} \mathrm{~K}_{120} \mathrm{~kg} / \mathrm{ha}\right)$.

Table 2. Effects of $\mathbf{N}$ and $\mathrm{K}$ on vegetative growth of gladiolus from cormel.

\begin{tabular}{|c|c|c|c|c|c|c|}
\hline \multirow[t]{3}{*}{ Treat } & \multicolumn{2}{|c|}{ Treatment combinations } & \multirow{3}{*}{$\begin{array}{l}\text { Leaf } \\
\text { length } \\
(\mathrm{cm})\end{array}$} & \multirow{3}{*}{$\begin{array}{l}\text { Leaf breadth } \\
\qquad(\mathrm{cm})\end{array}$} & \multirow{3}{*}{$\begin{array}{l}\text { Plant height } \\
\text { (cm) }\end{array}$} & \multirow{3}{*}{$\begin{array}{l}\text { Spikes } \\
(\%)\end{array}$} \\
\hline & $\mathrm{N}$ & $\mathrm{K}$ & & & & \\
\hline & \multicolumn{2}{|c|}{$\mathrm{kg} / \mathrm{ha}$} & & & & \\
\hline $\mathrm{T}_{1}$ & 0 & 120 & 34.3 & $1.61 \mathrm{~cd}$ & $39.8 \mathrm{bc}$ & $61.1 \mathrm{c}$ \\
\hline $\mathrm{T}_{2}$ & 50 & 120 & 35.0 & $1.70 \mathrm{bcd}$ & $40.4 \mathrm{ab}$ & $65.9 \mathrm{bc}$ \\
\hline $\mathrm{T}_{3}$ & 100 & 120 & 35.2 & $1.70 \mathrm{bcd}$ & $40.9 \mathrm{ab}$ & $72.2 \mathrm{bc}$ \\
\hline $\mathrm{T}_{4}$ & 150 & 120 & 35.7 & $1.73 \mathrm{bcd}$ & $40.9 \mathrm{ab}$ & $73.8 \mathrm{bc}$ \\
\hline $\mathrm{T}_{5}$ & 200 & 120 & 36.2 & $1.75 \mathrm{bc}$ & $41.4 \mathrm{ab}$ & $79.4 \mathrm{ab}$ \\
\hline $\mathrm{T}_{6}$ & 250 & 120 & 36.6 & $1.81 \mathrm{ab}$ & $41.9 \mathrm{a}$ & $80.2 \mathrm{ab}$ \\
\hline $\mathrm{T}_{7}$ & 150 & 0 & 34.9 & $1.64 \mathrm{bcd}$ & $40.8 \mathrm{ab}$ & $65.9 \mathrm{bc}$ \\
\hline $\mathrm{T}_{8}$ & 150 & 40 & 35.2 & $1.71 \mathrm{bcd}$ & $40.9 \mathrm{ab}$ & $68.3 \mathrm{bc}$ \\
\hline $\mathrm{T}_{9}$ & 150 & 80 & 35.6 & $1.73 \mathrm{bcd}$ & $41.0 \mathrm{ab}$ & $72.2 \mathrm{bc}$ \\
\hline $\mathrm{T}_{10}$ & 150 & 160 & 36.2 & $1.74 \mathrm{bc}$ & $41.8 \mathrm{a}$ & $78.6 \mathrm{ab}$ \\
\hline $\mathrm{T}_{11}$ & 150 & 200 & 36.4 & $1.93 \mathrm{a}$ & $42.1 \mathrm{a}$ & $88.1 \mathrm{a}$ \\
\hline $\mathrm{T}_{12}$ & Native c & & 32.7 & $1.55 \mathrm{~d}$ & $38.4 \mathrm{c}$ & $45.2 \mathrm{~d}$ \\
\hline \multicolumn{3}{|c|}{ Level of significance } & NS & $* *$ & $* *$ & ** \\
\hline \multicolumn{3}{|l|}{ CV (\%) } & 4.19 & 4.27 & 1.86 & 6.51 \\
\hline
\end{tabular}

Means with the same letter(s) are not significantly different at $1 \%$ by DMRT **, Significant at $1 \%$ level; NS, Not significant

Spikes: Significant effect was also observed in spikes percent (Table 2). The highest percentage of spikes $(88.1 \%)$ were produced by the treatment $T_{11}\left(\mathrm{~N}_{150}\right.$ $\mathrm{K}_{200} \mathrm{~kg} / \mathrm{ha}$ ), which was statistically similar to $\mathrm{T}_{5}, \mathrm{~T}_{6}$, and $\mathrm{T}_{10}$, whereas control treatment produced the lowest spike percentage (45.2\%)

\section{Effects of $\mathbf{N}$ and $\mathrm{K}$ on corm production of gladiolus}

Significant variation was observed in corm production due to different levels of $\mathrm{N}$ and $\mathrm{K}$ (Table 3 ).

Corm production: The maximum percentage of corm (97.6\%) was produced by the treatment combination $\mathrm{T}_{11}\left(\mathrm{~N}_{150} \mathrm{~K}_{200} \mathrm{~kg} / \mathrm{ha}\right)$, which was statistically different 
from other treatments indicating an optimum dose of $\mathrm{N}$ and K. Statistically no difference was observed among the treatment started from $T_{1}$ to $T_{10}$. The lowest percentage of corm (61.1\%) was observed with control (Table 3 ).

Table 3. Effects of $\mathbf{N}$ and $\mathbf{K}$ on corm production of gladiolus from cormel.

\begin{tabular}{|c|c|c|c|c|c|c|c|}
\hline \multirow{3}{*}{$\begin{array}{l}\text { Tr. } \\
\text { no. }\end{array}$} & \multicolumn{2}{|c|}{$\begin{array}{c}\text { Treatment } \\
\text { combinations }\end{array}$} & \multirow[t]{3}{*}{$\begin{array}{c}\text { Corms } \\
(\%)\end{array}$} & \multirow{3}{*}{$\begin{array}{c}\text { Corm } \\
\text { weight } \\
\text { (g) }\end{array}$} & \multirow{3}{*}{$\begin{array}{l}\text { Corm } \\
\text { diamet } \\
\text { er } \\
(\mathrm{cm})\end{array}$} & \multirow{3}{*}{$\begin{array}{c}\text { Flowering } \\
\text { sized } \\
\text { corm } \\
(\geq 2.5 \mathrm{~cm}) \\
(\%) \\
\end{array}$} & \multirow{3}{*}{$\begin{array}{c}\text { Corm } \\
\text { number/ } \\
\text { ha } \\
(000)\end{array}$} \\
\hline & $\mathrm{N}$ & $\mathrm{K}$ & & & & & \\
\hline & \multicolumn{2}{|c|}{$\mathrm{kg} / \mathrm{ha}$} & & & & & \\
\hline $\mathrm{T}_{1}$ & 0 & 120 & $\begin{array}{c}84.1 \\
(64.4) \mathrm{b}\end{array}$ & $14.2 \mathrm{bcd}$ & $3.38 \mathrm{~b}$ & $\begin{array}{c}91.5 \\
(70.5) \mathrm{c}\end{array}$ & $104 \mathrm{bc}$ \\
\hline $\mathrm{T}_{2}$ & 50 & 120 & $\begin{array}{c}84.9 \\
(64.9) \mathrm{b}\end{array}$ & $14.3 \mathrm{bcd}$ & $3.42 \mathrm{~b}$ & $\begin{array}{c}93.5 \\
(72.6) b c\end{array}$ & $105 \mathrm{bc}$ \\
\hline $\mathrm{T}_{3}$ & 100 & 120 & $\begin{array}{c}85.7 \\
(65.5) b\end{array}$ & $15.0 \mathrm{bc}$ & $3.54 \mathrm{~b}$ & $\begin{array}{c}94.5 \\
(74.0) b c\end{array}$ & $106 \mathrm{abc}$ \\
\hline $\mathrm{T}_{4}$ & 150 & 120 & $\begin{array}{c}86.5 \\
(66.4) \mathrm{b}\end{array}$ & $15.3 \mathrm{bc}$ & $3.58 \mathrm{~b}$ & $\begin{array}{c}95.5 \\
(75.1) b c\end{array}$ & $107 \mathrm{abc}$ \\
\hline $\mathrm{T}_{5}$ & 200 & 120 & $\begin{array}{c}88.9 \\
(68.1) b\end{array}$ & $16.0 \mathrm{bc}$ & $3.63 \mathrm{~b}$ & $\begin{array}{c}96.5 \\
(76.6) \mathrm{b}\end{array}$ & $109 \mathrm{ab}$ \\
\hline $\mathrm{T}_{6}$ & 250 & 120 & $\begin{array}{c}88.9 \\
(68.3) b\end{array}$ & $16.2 \mathrm{~b}$ & $3.64 \mathrm{~b}$ & $\begin{array}{c}96.6 \\
(76.8) b\end{array}$ & $109 \mathrm{ab}$ \\
\hline $\mathrm{T}_{7}$ & 150 & 0 & $\begin{array}{c}75.4 \\
(58.3) \mathrm{bc}\end{array}$ & $13.4 \mathrm{~cd}$ & $3.03 \mathrm{c}$ & $\begin{array}{c}79.2 \\
(60.7) \mathrm{d}\end{array}$ & $93 \mathrm{c}$ \\
\hline $\mathrm{T}_{8}$ & 150 & 40 & $\begin{array}{c}84.9 \\
(64.8) b\end{array}$ & $14.3 \mathrm{bcd}$ & $3.40 \mathrm{~b}$ & $\begin{array}{c}93.4 \\
(72.6) \mathrm{bc}\end{array}$ & $105 \mathrm{bc}$ \\
\hline $\mathrm{T}_{9}$ & 150 & 80 & $\begin{array}{c}85.7 \\
(65.6) b\end{array}$ & $14.7 \mathrm{bcd}$ & $3.45 \mathrm{~b}$ & $\begin{array}{c}94.5 \\
(74.0) \text { bc }\end{array}$ & $106 \mathrm{abc}$ \\
\hline $\mathrm{T}_{10}$ & 150 & 160 & $\begin{array}{c}88.9 \\
(68.5) b\end{array}$ & $16.5 \mathrm{~b}$ & $3.75 \mathrm{~b}$ & $\begin{array}{c}97.3 \\
(77.7) b\end{array}$ & $109 \mathrm{ab}$ \\
\hline $\mathrm{T}_{11}$ & 150 & 200 & $\begin{array}{c}97.6 \\
(79.9) \mathrm{a}\end{array}$ & $19.5 \mathrm{a}$ & $4.11 \mathrm{a}$ & $\begin{array}{c}100.0 \\
(86.0) \mathrm{a}\end{array}$ & $120 \mathrm{a}$ \\
\hline $\mathrm{T}_{12}$ & Nati & & $\begin{array}{c}61.1 \\
(49.6) \mathrm{c}\end{array}$ & $12.2 \mathrm{~d}$ & $2.80 \mathrm{c}$ & $\begin{array}{c}58.5 \\
(48.2) \mathrm{e}\end{array}$ & $75 \mathrm{~d}$ \\
\hline Lev & 1 of $s$ & & $* *$ & $* *$ & $* *$ & $* *$ & $* *$ \\
\hline $\mathrm{CV}$ & & & 6.36 & 6.59 & 4.27 & 2.93 & 5.69 \\
\hline
\end{tabular}

Means with the same letter(s) are not significantly different at $1 \%$ by DMRT $* *$, Significant at $1 \%$ level

Figures in parentheses are transformed values

Corm weight: The heaviest corm (19.5 g) was obtained with $\mathrm{T}_{11}$ treatment $\left(\mathrm{N}_{150}\right.$ $\mathrm{K}_{200} \mathrm{~kg} / \mathrm{ha}$ ), which was statistically different from the rest of the treatments. In an experiment, Das (1998) reported that the corms weight/plant was significantly 
higher with increasing dose of $\mathrm{K}\left(200 \mathrm{~kg} \mathrm{~K}_{2} \mathrm{O} / \mathrm{ha}\right)$. Other treatments showed statistically similar trends $(14.2 \mathrm{~g}-16.5 \mathrm{~g})$ except control and $\mathrm{T}_{7}$ (Table 3$)$. The lightest corm was produced by control (12.2 g).

Corm diameter: Treatment $\mathrm{T}_{11}\left(\mathrm{~N}_{150} \mathrm{~K}_{200} \mathrm{~kg} / \mathrm{ha}\right)$ produced the largest corm (4.11 $\mathrm{cm})$, which was statistically different from other treatments. The smallest corm was obtained in control $\left(2.80 \mathrm{~cm}\right.$ ) followed by $\mathrm{T}_{7}$, which contained no $\mathrm{K}$ (Table $3)$. For corm development, $\mathrm{K}$ supply is very much important. According to Misra and Singh (1998), the effect of N was enhanced when K was added. Mukherjee et al. (1994) found the largest corms with $50 \mathrm{~g} \mathrm{~N}$ and $20 \mathrm{~g} \mathrm{~K} / \mathrm{m}^{2}$, which was equivalent to $500 \mathrm{~kg} \mathrm{~N}$ and $200 \mathrm{~kg} \mathrm{~K} / \mathrm{ha}$.

Flowering sized corm: From the Table 3, it was found that the cent percent flowering sized corms were produced from cormel due to the $\mathrm{T}_{11}$ treatment $\left(\mathrm{N}_{150}\right.$ $\mathrm{K}_{200} \mathrm{~kg} / \mathrm{ha}$ ). The control treatment received only $58.5 \%$ flowering sized corm. Treatment $\mathrm{T}_{7}\left(\mathrm{~N}_{150} \mathrm{~K}_{0} \mathrm{~kg} / \mathrm{ha}\right)$ also showed lower performances in respect of flowering sized corm $(79.2 \%)$. This indicate the effect of $\mathrm{N}$ was enhanced when K was applied (Misra and Singh, 1998).

Corm yield: Treatment $\mathrm{T}_{11}\left(\mathrm{~N}_{150} \mathrm{~K}_{200} \mathrm{~kg} / \mathrm{ha}\right)$ produced the maximum number of corms $(120,000)$, which was statistically similar to $T_{10}, T_{6}, T_{5}, T_{4}, T_{3}$, and $T_{9}$ (Table 3$)$. The control treatment had the minimum numbers/ha $(75,000)$. The results indicate that an optimum fertilizer dose encouraged plants to grow more vigorously and produce more metabolites.

\section{Effects of $\mathbf{N}$ and $\mathrm{K}$ on cormel production of gladiolus}

Cormel production from cormel showed significant differences among the treatments for the various levels of $\mathrm{N}$ and $\mathrm{K}$ (Table 4).

Cormels/hill: The maximum number (12.4) of cormels/hill was produced by $\mathrm{T}_{11}$ $\left(\mathrm{N}_{150} \mathrm{~K}_{200} \mathrm{~kg} / \mathrm{ha}\right)$ followed by $\mathrm{T}_{10}\left(\mathrm{~N}_{150} \mathrm{~K}_{160} \mathrm{~kg} / \mathrm{ha}\right)$. The minimum number of cormels/hill was recorded in control followed by $\mathrm{T}_{7}\left(\mathrm{~N}_{150} \mathrm{~K}_{0} \mathrm{~kg} / \mathrm{ha}\right)$, which contained no K (Table 4).

Cormel weight/hill: Treatment $T_{11}\left(\mathrm{~N}_{150} \mathrm{~K}_{200} \mathrm{~kg} / \mathrm{ha}\right)$ demonstrated that the highest cormel weight $(16.3 \mathrm{~g})$ per hill, which was statistically superior to other treatments. The lowest weight was recorded in control treatment $(5.17 \mathrm{~g})$.

Cormel yield: The maximum cormel yield $\left(1.66 \mathrm{t}\right.$ ) was found in treatment $T_{11}$ $\left(\mathrm{N}_{150} \mathrm{~K}_{200} \mathrm{~kg} / \mathrm{ha}\right)$, which was statistically superior to other treatments. The lowest yield was produced by control $(0.43 \mathrm{t} / \mathrm{ha})$. Treatment $\mathrm{T}_{7}$ also showed statistically lower yield (0.81 t/ha), which contained no K (Table 4). Misra and Singh (1998) reported that balanced fertilizer, particularly with $\mathrm{K}$, resulted in an increase in yield of cormels. 
Table 4. Effects of $\mathbf{N}$ and $\mathbf{K}$ on cormel production of gladiolus from cormel.

\begin{tabular}{|c|c|c|c|c|c|}
\hline \multirow[t]{3}{*}{ Treat } & \multicolumn{2}{|c|}{ Treatment combinations } & \multirow{3}{*}{$\begin{array}{l}\text { Cormels/ } \\
\text { hill }\end{array}$} & \multirow{3}{*}{$\begin{array}{l}\text { Cormel weight/ } \\
\text { hill (g) }\end{array}$} & \multirow{3}{*}{$\begin{array}{c}\text { Cormel } \\
\text { yield/ha (t) }\end{array}$} \\
\hline & $\mathrm{N}$ & $\mathrm{K}$ & & & \\
\hline & \multicolumn{2}{|c|}{$\mathrm{kg} / \mathrm{ha}$} & & & \\
\hline $\mathrm{T}_{1}$ & 0 & 120 & $8.30 \mathrm{de}$ & $8.17 \mathrm{de}$ & $0.91 \mathrm{fg}$ \\
\hline $\mathrm{T}_{2}$ & 50 & 120 & 8.50 cde & $9.50 \mathrm{~cd}$ & $1.0 \mathrm{ef}$ \\
\hline $\mathrm{T}_{3}$ & 100 & 120 & 8.87 bcde & $9.67 \mathrm{~cd}$ & $1.10 \mathrm{de}$ \\
\hline $\mathrm{T}_{4}$ & 150 & 120 & 9.10 bcde & $10.7 \mathrm{bc}$ & $1.23 \mathrm{~cd}$ \\
\hline $\mathrm{T}_{5}$ & 200 & 120 & $10.3 \mathrm{bcd}$ & $12.2 \mathrm{~b}$ & $1.38 \mathrm{bc}$ \\
\hline $\mathrm{T}_{6}$ & 250 & 120 & $10.4 \mathrm{bc}$ & $12.3 \mathrm{~b}$ & $1.41 \mathrm{~b}$ \\
\hline $\mathrm{T}_{7}$ & 150 & 0 & $7.10 \mathrm{ef}$ & $7.17 \mathrm{e}$ & $0.81 \mathrm{~g}$ \\
\hline $\mathrm{T}_{8}$ & 150 & 40 & $8.50 \mathrm{cde}$ & $8.83 \mathrm{cde}$ & $0.92 \mathrm{fg}$ \\
\hline $\mathrm{T}_{9}$ & 150 & 80 & 8.67 bcde & $9.50 \mathrm{~cd}$ & $1.06 \mathrm{ef}$ \\
\hline $\mathrm{T}_{10}$ & 150 & 160 & $10.6 \mathrm{ab}$ & $12.4 \mathrm{~b}$ & $1.44 \mathrm{~b}$ \\
\hline $\mathrm{T}_{11}$ & 150 & 200 & $12.4 \mathrm{a}$ & $16.3 \mathrm{a}$ & $1.66 \mathrm{a}$ \\
\hline $\mathrm{T}_{12}$ & \multicolumn{2}{|c|}{ Native control } & $5.37 \mathrm{f}$ & $5.17 \mathrm{f}$ & $0.43 \mathrm{~h}$ \\
\hline \multicolumn{3}{|c|}{ Level of significance } & $* *$ & $* *$ & $* *$ \\
\hline \multicolumn{3}{|l|}{ CV (\%) } & 8.78 & 8.10 & 6.35 \\
\hline
\end{tabular}

Means with the same letter(s) are not significantly different at $1 \%$ level by DMRT

**, Significant at $1 \%$ level

Grades of cormels: The treatments exhibited significant variations in respect of different grades of cormels by number (Fig. 1). The highest percentage $(6.19 \%)$ of large grade cormels $(>2.0 \mathrm{~cm})$ was produced by the $\mathrm{T}_{11}$ treatment $\left(\mathrm{N}_{150} \mathrm{~K}_{200}\right.$ $\mathrm{kg} / \mathrm{ha}$ ) followed by $\mathrm{T}_{10}(4.22 \%)$. On the other hand, no large grade cormel was recorded in $\mathrm{T}_{7}\left(\mathrm{~N}_{150} \mathrm{~K}_{0} \mathrm{~kg} / \mathrm{ha}\right)$ and Control. Similarly, the maximum percentage of medium grade cormels was found in treatments $\mathrm{T}_{11}$ and $\mathrm{T}_{10}(90.29 \%$ and $90.28 \%$, respectively) and the minimum was found in control (66.2\%). Opposite results were found in case of small grade cormels. The lowest number of small grade cormels $(3.52 \%)$ was recorded by $\mathrm{T}_{11}$ and the highest number $(33.8 \%)$ was registered in control. Better sized cormels were produced by the application of higher dose of $\mathrm{N}(375 \mathrm{~kg} / \mathrm{ha})$ and $\mathrm{K}(250 \mathrm{~kg} / \mathrm{ha})$ when corm was used as seed materials (Sultana et al., 2005). 


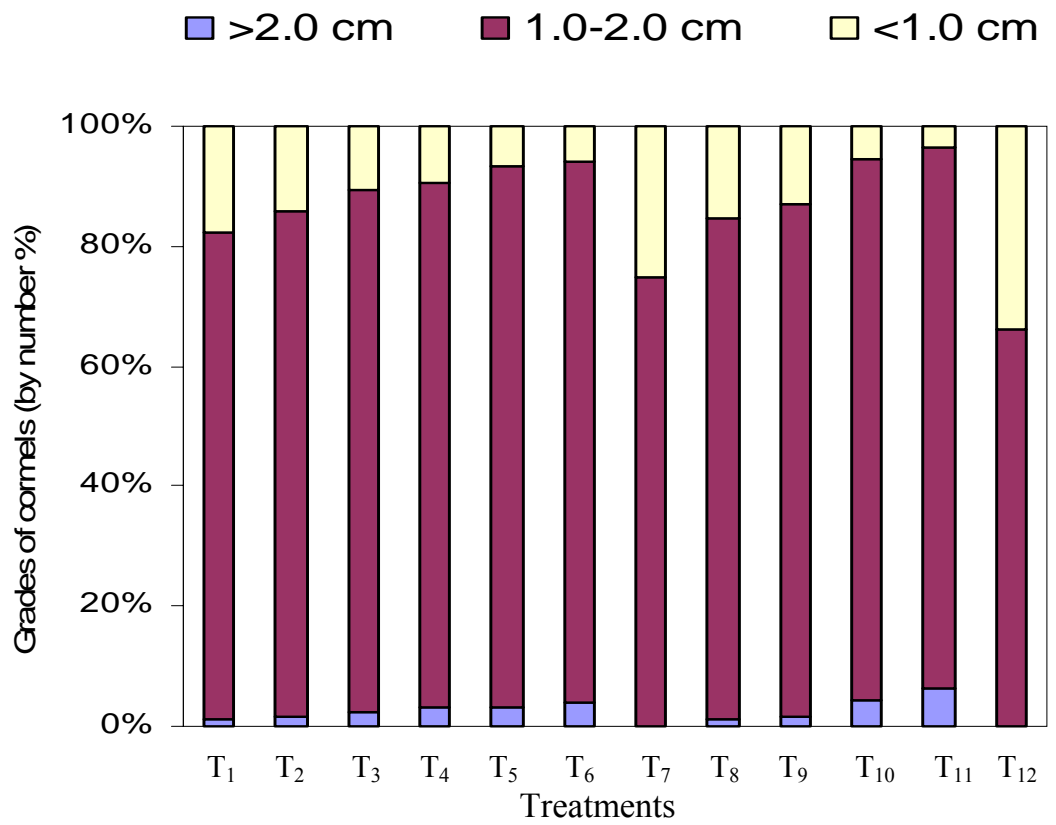

Fig. 1. Percentage of grades of cormels as influenced by different levels of $\mathrm{N}$ and $\mathrm{K}$. Treatment details have been shown in Table 1.

\section{Effects of $\mathbf{N}$ and $\mathrm{K}$ on flower production of gladiolus}

Flower production also significantly influenced by the corms produced in the previous year due to the effect of $\mathrm{N}$ and $\mathrm{K}$ (Table 5).

Florets/spike: The maximum number of florets/spike (13.1) was produced by the corms treated with the treatment combination of $\mathrm{N}_{150} \mathrm{~K}_{200} \mathrm{~kg} / \mathrm{ha}\left(\mathrm{T}_{11}\right)$, which was statistically identical with other treatments except $\mathrm{T}_{7}\left(\mathrm{~N}_{150} \mathrm{~K}_{0} \mathrm{~kg} / \mathrm{ha}\right)$ and control (Table 5). Sidhu and Arora (1989) found the maximum florets/spike when $\mathrm{N}$ was applied at $200 \mathrm{~kg} / \mathrm{ha}$. Kosugi and Kondu (1960) reported that where $\mathrm{N}$ had been supplied in the previous year, the number of florets/spike was increased in the next year.

Spike length: The longest spike $(82.2 \mathrm{~cm})$ was produced by the treatment combination of $T_{11}\left(N_{150} K_{200} \mathrm{~kg} / \mathrm{ha}\right)$, which was statistically similar to $T_{10}, T_{6}$, $\mathrm{T}_{5}$, and $\mathrm{T}_{4}$. The Control treatment received the shortest spike $(74.3 \mathrm{~cm})$ followed by $\mathrm{T}_{7}$. Sidhu and Arora (1989) reported that spike length was significantly improved by the application of $20 \mathrm{~g} \mathrm{~N} / \mathrm{m}^{2}$ which is equivalent to $200 \mathrm{~kg} \mathrm{~N} / \mathrm{ha}$. 
Table 5. Performance of flower production of gladiolus from corms produced in the previous year due to the effect of $\mathrm{N}$ and $\mathrm{K}$.

\begin{tabular}{|c|c|c|c|c|c|c|c|}
\hline \multirow[t]{3}{*}{ Treat. } & \multicolumn{2}{|c|}{$\begin{array}{c}\text { Treatment } \\
\text { combinations }\end{array}$} & \multirow{3}{*}{$\begin{array}{l}\text { Florets/ } \\
\text { spike }\end{array}$} & \multirow{3}{*}{$\begin{array}{c}\text { Spike } \\
\text { length } \\
(\mathrm{cm})\end{array}$} & \multirow{3}{*}{$\begin{array}{l}\text { Rachis } \\
\text { length } \\
(\mathrm{cm})\end{array}$} & \multirow{3}{*}{$\begin{array}{c}\text { Flower } \\
\text { stick } \\
\text { weight }(g)\end{array}$} & \multirow[t]{3}{*}{$\begin{array}{c}\text { Flower } \\
\text { stick }(\%)\end{array}$} \\
\hline & $\mathrm{N}$ & $\mathrm{K}$ & & & & & \\
\hline & \multicolumn{2}{|c|}{$\mathrm{kg} / \mathrm{ha}$} & & & & & \\
\hline $\mathrm{T}_{1}$ & 0 & 120 & $12.4 \mathrm{ab}$ & $78.3 \mathrm{~cd}$ & $41.8 \mathrm{bc}$ & $49.4 \mathrm{ab}$ & 82 ef \\
\hline $\mathrm{T}_{2}$ & 50 & 120 & $12.6 \mathrm{ab}$ & $78.8 \mathrm{bcd}$ & $42.2 \mathrm{abc}$ & $52.2 \mathrm{ab}$ & 82 ef \\
\hline $\mathrm{T}_{3}$ & 100 & 120 & $12.6 \mathrm{ab}$ & $79.4 \mathrm{bc}$ & $42.4 \mathrm{abc}$ & $52.6 \mathrm{ab}$ & $93 \mathrm{~cd}$ \\
\hline $\mathrm{T}_{4}$ & 150 & 120 & $12.5 \mathrm{ab}$ & $79.7 \mathrm{abc}$ & $42.7 \mathrm{abc}$ & $53.1 \mathrm{ab}$ & $95 \mathrm{bc}$ \\
\hline $\mathrm{T}_{5}$ & 200 & 120 & $12.6 \mathrm{ab}$ & $80.5 \mathrm{abc}$ & $43.8 \mathrm{ab}$ & $55.4 \mathrm{a}$ & $103 \mathrm{abc}$ \\
\hline $\mathrm{T}_{6}$ & 250 & 120 & $12.7 \mathrm{ab}$ & $81.0 \mathrm{abc}$ & $43.8 \mathrm{ab}$ & $55.5 \mathrm{a}$ & $103 a b c$ \\
\hline $\mathrm{T}_{7}$ & 150 & 0 & $12.0 \mathrm{bc}$ & $76.3 \mathrm{de}$ & $39.9 \mathrm{~cd}$ & $46.0 \mathrm{ab}$ & $72 \mathrm{fg}$ \\
\hline $\mathrm{T}_{8}$ & 150 & 40 & $12.6 \mathrm{ab}$ & $78.4 \mathrm{~cd}$ & $42.1 \mathrm{abc}$ & $52.0 \mathrm{ab}$ & $83 \mathrm{de}$ \\
\hline $\mathrm{T}_{9}$ & 150 & 80 & $12.6 \mathrm{ab}$ & $79.5 \mathrm{bc}$ & $42.4 \mathrm{abc}$ & $52.3 \mathrm{ab}$ & $93 \mathrm{bcd}$ \\
\hline $\mathrm{T}_{10}$ & 150 & 160 & $13.0 \mathrm{a}$ & $81.5 \mathrm{ab}$ & $44.5 \mathrm{ab}$ & $56.3 \mathrm{a}$ & $105 \mathrm{ab}$ \\
\hline $\mathrm{T}_{11}$ & 150 & 200 & $13.1 \mathrm{a}$ & $82.2 \mathrm{a}$ & $45.4 \mathrm{a}$ & $57.2 \mathrm{a}$ & $113 \mathrm{a}$ \\
\hline $\mathrm{T}_{12}$ & \multicolumn{2}{|c|}{ Native control } & $11.5 \mathrm{c}$ & $74.3 \mathrm{e}$ & $38.3 \mathrm{~d}$ & $42.8 \mathrm{~b}$ & $65 \mathrm{~g}$ \\
\hline \multicolumn{3}{|c|}{ Level of significance } & $*$ & $* *$ & $* *$ & $* *$ & $* *$ \\
\hline \multicolumn{3}{|c|}{ CV $(\%)$} & 3.64 & 1.35 & 3.01 & 8.10 & 5.05 \\
\hline
\end{tabular}

Means with the same letter(s) are not significantly different at $1 \%$ and $5 \%$ level by DMRT

**, Significant at $1 \%$ level *, Significant at $5 \%$ level

Rachis length: Treatment $T_{11}\left(\mathrm{~N}_{150} \mathrm{~K}_{200} \mathrm{~kg} / \mathrm{ha}\right)$ produced the longest rachis (45.4 $\mathrm{cm}$ ), which was statistically identical to all other treatments except $T_{1}, T_{7}$ and control treatments. The shortest rachis $(38.3 \mathrm{~cm})$ was produced by control treatment followed by $\mathrm{T}_{7}(39.9 \mathrm{~cm})$. A balanced fertilizer is essential for better performance. Excess $\mathrm{N}$, absence of adequate $\mathrm{K}$ is unfavorable to flower quality due to nutritional imbalance (Mukhopadhaya, 1995).

Flower stick weight: The heaviest flower stick $(57.2 \mathrm{~g})$ was produced by the corms obtained through the treatment combinations of $\mathrm{N}_{150} \mathrm{~K}_{200} \mathrm{~kg} / \mathrm{ha}\left(\mathrm{T}_{11}\right)$, which was statistically similar with all other treatments except control. According to Litterell and Waters (1967) $200 \mathrm{~kg} / \mathrm{ha}$ of $\mathrm{N}$ increased the weight of the spikes produced in the second season. Corms of control treatment produced the lightest flower stick (42.8 g).

Flower sticks: Percentage of flower sticks showed significant variations among the treatments due to the effect of $\mathrm{N}$ and $\mathrm{K}$ fertilizer (Table 5). The maximum flower sticks (113\%) were recorded with the treatment $T_{11}\left(\mathrm{~N}_{150} \mathrm{~K}_{200} \mathrm{~kg} / \mathrm{ha}\right)$, which was statistically identical with treatments $T_{10}, T_{6}$, and $T_{5}$. The lowest flower sticks $(65 \%)$ were recorded in control followed by $\mathrm{T}_{7}$ treatment. It appears 
that the corms which performed better in the previous year, they also showed better performances in flower production.

\section{Conclusion}

Overall results indicate that the treatment combination $\mathrm{N}_{150} \mathrm{~K}_{200} \mathrm{~kg} / \mathrm{ha}$ is the optimum for the production of gladiolus corm and cormel when cormels were used as planting materials.

\section{References}

Das, T.K. 1998. Corm and cormel production in gladiolus as affected by spike removal and K application. Indian J. Hort. 55(4):327-331.

Kosugi, K. and M. Kondo. 1960. Studies on blindness in gladiolus VII. Effect of fertilizer treatment in the previous year on flowering in the current year. J. Hort. Ass. Japan 29:163-168.

Litterell, R.H. and W.E. Waters. 1967. Influence of nitrogen and lime fertilization on gladiolus corms and flower production and internal microflora of corms. Proceedings of the Florida State Horticultural Society. 80:405-408.

Misra, R.L. and B. Singh. 1998. Gladiolus. In: Commercial Flowers. (Eds) Bose, T. K. and L. P. Yadav. Naya Prokash, Calcutta, India. pp. 266-353.

Mukherjee, S., S.C. Jana and T.K. Chatterjee. 1994. Effect of nitrogen and phosphorus doses on production of flower and corms of gladiolus. Indian Agriculturist. 38(3):211-213.

Mukhopadhyay, A. 1995. Gladiolus. Publication and Information Division. Indian Council of Agric. Res., Krishi Anusandhan Bhavan, New Delhi. pp.1-83.

Shah, A., S. D. Lal and J. N. Seth. 1984. Effect of different levels of nitrogen and phosphorus on growth, flowering and corm yield of gladiolus cv. Vinks Glory. Progressive Horticulture. 16(3/4): 305-307.

Sidhu, G.S. and J.S. Arora. 1989. Response of gladiolus varieties to nitrogen application. Indian J. Hort. 42(2):250-54.

Sultana, S., F.N. Khan, A.T.M. Farid, M.S. Mollah and M.A. Haque. 2005. Nutrient management for gladiolus. Bangladesh J. Agric. and Environ. 1(2): 7-12.

Sultana, S., F.N. Khan, M.A. Haque, S. Akhter and S. Noor. 2006. Effect of NPK on growth and flowering in tuberose. J. Subtrop. Agric. Res. Dev. 4(2):111-113. 